\title{
BMJ Open Effects of oil-soluble versus water- soluble contrast media at hysterosalpingography on pregnancy outcomes in women with a low risk of tubal disease: study protocol for a randomised controlled trial
}

\author{
Guiling Liang, ${ }^{1,2,3}$ Qian Zhu, ${ }^{1,2,3}$ Xiaoqing He, ${ }^{1,2,3}$ Xiaofeng Wang, ${ }^{1,2,3}$ Ling Jiang, ${ }^{2,3,4}$ \\ Chenfeng Zhu, ${ }^{1,2,3}$ Li Xie (D) , ${ }^{5}$ Zhaoxia Qian, ${ }^{2,3,4}$ Jian Zhang (1) ${ }^{1,2,3}$
}

To cite: Liang G, Zhu Q, He X, et al. Effects of oil-soluble versus water-soluble contrast media at hysterosalpingography on pregnancy outcomes in women with a low risk of tubal disease: study protocol for a randomised controlled trial. BMJ Open 2020;10:e039166. doi:10.1136/ bmjopen-2020-039166

- Prepublication history for this paper is available online. To view these files, please visit the journal online (http://dx.doi. org/10.1136/bmjopen-2020039166).

Received 07 April 2020 Revised 30 August 2020 Accepted 04 September 2020

Check for updates

(c) Author(s) (or their employer(s)) 2020. Re-use permitted under CC BY-NC. No commercial re-use. See rights and permissions. Published by BMJ.

For numbered affiliations see end of article.

Correspondence to

Zhaoxia Qian;

zhaoxiaqian@163.com and

Jian Zhang;

zhangjian_sjtu@126.com

\section{ABSTRACT}

Introduction In recent years, due to various factors, the rate of infertility in China has increased and now affects over $10 \%$ of women of reproductive age. Hysterosalpingography (HSG) is a common diagnostic procedure during fertility examinations. However, there is no consensus on the choice of contrast agents and their effects. As the largest multicentre, randomised controlled trial (H2Oil trial from the Netherlands) has shown that oilsoluble contrast at HSG can enhance fertility compared with water-soluble contrast, we propose this study to examine whether the use of oil-soluble contrast media results in increased rates of pregnancy in Chinese women undergoing HSG.

Methods and analysis This study is a single-centre, randomised, controlled, parallel-group, superiority trial. Patients with low risk of tubal disease will be randomised to undergo HSG using iodinated oil injection (OSCM group, oil-soluble contrast media) or ioversol injection (WSCM group, water-soluble contrast media). To evaluate the potential superiority of the OSCM group, with 1:1 allocation ratio, $90 \%$ statistical power and a two-sided significance level of $5 \%$, we have calculated a sample of 520 women per group to be enrolled, for a total of 1040 including $10 \%$ loss to follow-up or protocol variation. The primary outcome is the rate of ongoing pregnancy during 6 months after randomisation. The secondary outcomes will consist of thyroid function of patients and newborns, pain scores during HSG, rate of live birth, clinical pregnancies, miscarriages, ectopic pregnancy, time to ongoing pregnancy, time to live birth, cost calculations of the OSCM group/WSCM group, and assisted reproductive technology treatments between the two groups.

Ethics and dissemination This protocol received authorisation from the Medical Research Ethics Committee of International Peace Maternity and Child Health Hospital on 18 January 2020 (approval no GKLW2020-02). The findings will be reported in peer-reviewed publications and presentations at international scientific meetings. Trial registration number ChiCTR2000031612.
Strengths and limitations of this study

This is a study designed to assess the thyroid func tion of patients at different time points who undergo hysterosalpingography (HSG) and their newborns' thyroid function in a randomised controlled trial (RCT).

- This is a study with long-term follow-up of patients who undergo HSG in an RCT which may explore cumulative pregnancy rate and cumulative live birth.

- This study will record pain intensity and when pain occurs during HSG.

- The trial is based in a single centre, which might limit the generalisability of the findings.

- The trial will not be blinded with respect to participants and caregivers.

\section{INTRODUCTION}

Infertility is defined as the failure to establish pregnancy after 12 months of regular, unprotected sexual intercourse or an impairment of a person's capacity to reproduce either as an individual or with his/her partner. ${ }^{1}$ Due to the enactment of the second child policy in China, the increasing age of mothers, environmental pollution and workplace pressures, the rate of infertility in China has increased and now affects more than $10 \%$ of reproductive women. ${ }^{2}$

Hysterosalpingography (HSG) refers to the radiographic evaluation of the uterine cavity and the Fallopian tubes after the injection of an oil-soluble/water-soluble contrast medium through the cervical canal. ${ }^{3}$ HSG plays an important role in the evaluation of female Fallopian tube reproductive function and is a common diagnostic procedure during fertility examinations. ${ }^{45}$ In China, it is the preferred 
method to investigate tubal patency. ${ }^{6}$ Compared with laparoscopy, it is less expensive and has fewer complications. ${ }^{7}$ In addition to diagnostic information, HSG has therapeutic effects which are associated with increased fecundability in the months after the procedure. ${ }^{89}$

Oil-soluble contrast media (OSCM) is represented by iodinated oil injection, which uses poppy seed oil as the raw material. It provides clear images and may have some anti-inflammatory effects that perhaps enhance fertility. ${ }^{10}$ Due to less absorption of the contrast media, the occurrence of adverse events is notably reduced. ${ }^{11} 12$ Water-soluble contrast media (WSCM) is represented by meglumine diatrizoate and ioversol. The advantages of WSCM are as follows: low viscosity, good fluidity, and rapid diffusion into the Fallopian tubes, which can clearly show their anatomy. However, the rapid flow may result in insufficient observation of Fallopian tube lesions and may result in poor Fallopian tube dredging. ${ }^{11}$

Some studies showed significantly higher rates of pregnancy after tubal flushing with OSCM. ${ }^{1314}$ This might be caused by flushing debris and dislodging mucus plugs from otherwise undamaged tubes. Additionally, OSCM might have an effect on peritoneal macrophage activity and on endometrial receptivity, thereby enhancing fertility. ${ }^{15}{ }^{16}$ Fang et al $\mathrm{s}^{17}$ systematic review, through a comprehensive analysis of six studies, showed that HSG using OSCM may promote the ongoing rate of pregnancy. However, the review did not include the Chinese population and did not report relevant content about thyroid function or long-term effects. In the largest multicentre, randomised controlled trial (RCT) to date, ${ }^{8}$ a total of 1119 women were randomly assigned to HSG with OSCM (557 women) or HSG with WSCM (562 women). The study, which was conducted in the Netherlands, showed higher ongoing pregnancy rates among women who underwent HSG with OSCM as compared with WSCM.

However, numerous studies have not shown a significant difference in rates of ongoing pregnancy between OSCM and WSCM groups. ${ }^{18-21}$ Lindequist $e t a t^{20}$ found that the rate of ongoing pregnancy in the OSCM group was slightly higher than the WSCM group, within 20 months after randomisation, but the difference was not statistically significant. In a multicentre RCT carried out by Spring et $a l^{21}{ }^{21}$ a total of 666 women were randomly assigned to WSCM group (260 women), OSCM group (273 women), or both WSCM and OSCM group (133 women). Differences in reproductive outcomes among the groups were not statistically significant.

Previous studies showed that women with subclinical hypothyroidism were more prone to OSCM-induced overt hypothyroidism, ${ }^{22}$ which may be due to the long half-life of OSCM excretion. ${ }^{23}$ It is known that excess iodine intake during pregnancy can adversely affect thyroid function in both the mother and the fetus. ${ }^{24}$ Nevertheless, there have been no large-scale, prospective studies exploring the relationship between HSG contrast agents and the occurrence of thyroid dysfunction in patients and neonates.
In summary, there is no consensus on the choice of contrast agents and their effects. In view of this uncertainty, we plan a single-centre, randomised, controlled, parallel-group, superiority trial among infertile Chinese women with a low a priori chance of tubal pathology.

\section{Objective and hypothesis}

The objective of the trial is to determine whether the use of OSCM during HSG results in a higher ongoing pregnancy rate compared with the use of WSCM for 6 months after randomisation. Our hypothesis is that in women undergoing HSG, the use of OSCM will result in a higher ongoing pregnancy rate compared with the use of WSCM.

\section{METHODS AND ANALYSIS \\ Study design and setting}

The study is a single-centre, randomised controlled, superiority trial and will be performed in the International Peace Maternity and Child Health Hospital, School of Medicine, Shanghai Jiao Tong University, Shanghai, China. The hospital has two experienced radiologists in HSG. The details of the study design are shown in figure 1 .

\section{Recruitment}

Before participation, patients need to complete a screening questionnaire. Eligible patients will be asked to participate in this study after receiving oral and written information from a gynaecologist in outpatient services when tubal testing has been indicated and will be scheduled. A research nurse will administer oral and written informed consent. To reach the target sample size, we will advertise our clinical trial using the hospital's official accounts on WeChat and distribute brochures in the outpatient department.

\section{Participants}

The inclusion criteria are as follows:

- Age 20-39 years old.

- Spontaneous menstrual cycles (cycle length between 25 and 35 days).

- Subfertility of at least 1 year and a fertile partner (defined as sperm count $>15 \times 10^{6}$ spermatozoa $/ \mathrm{mL}$ or a post-wash total motile sperm count $>3 \times 10^{6}$ spermatozoa $/ \mathrm{mL}$ before intrauterine insemination (IUI) )..$^{25}$

- Chlamydia trachomatis-negative via vaginal secretion culture (through chlamydia antigen detection) and no history of chlamydia infection.

- Low risk for tubal pathology according to medical history. (The patient has not been exposed to high risk factors of tubal pathology, such as history of chlamydia infection, pelvic inflammatory disease, known endometriosis or adenomyosis, pelvic abdominal surgery (including salpingostomy or salpingectomy for ectopic pregnancy and complicated appendectomy) and/or peritonitis.) $)^{26}$

- Valid indication for HSG in the fertility examination or before IUI treatment. 
- Signed informed consent.

The exclusion criteria are as follows:

- Irregular menstrual cycle, less than eight menstrual cycles per year.

- Endocrinopathological diseases, such as polycystic ovary syndrome, Cushing syndrome, adrenal hyperplasia, hyperprolactinaemia, acromegaly, hypothalamic amenorrhoea, hypothyroidy, diabetes mellitus and thyroid dysfunction.

- Known or at high risk for tubal pathology, $C$. trachomatis-positive of vaginal secretion culture and history of chlamydia infection.

- Known contrast (iodine) allergy.

- Male subfertility, defined as sperm count $<15 \times 10^{6}$ spermatozoa $/ \mathrm{mL}$ or a post-wash total motile sperm count $<3 \times 10^{6}$ spermatozoa $/ \mathrm{mL}$ before IUI.

- A contraindication to HSG.

- Not willing or able to sign the consent form.

\section{Randomisation}

Randomisation will take place on the day of HSG. Demographic, medical, gynaecological and infertility information will be collected at baseline. Patients will then be randomised between the two groups (OSCM or WSCM). Randomisation will be performed by an independent statistician using a web-based Research Electronic Data Capture (REDCap) system. The allocation ratio will be 1:1 and permuted blocks of 4-6 will be used. Prior to randomisation, screening data will be entered in the digital platform. The randomisation list will be sealed in sequentially numbered opaque envelopes. The envelopes will be stored in a double-locked cabinet and will only be opened by the practitioner to assign participants to each group after obtaining informed consent and having been screened for eligibility. Opened envelopes will again be separately stored in a double-locked cabinet.

\section{Blinding}

Owing to the difference in imaging between the use of OSCM and WSCM, and since the outcome measure of ongoing pregnancy will be an objective measure, the trial will not be blinded with respect to participants and caregivers.

\section{Intervention}

All patients will undergo HSG. Patients allocated to OSCM will undergo HSG with iodinated oil injection, a solution of Ethiodol that contains poppy seed oil and iodine (480 I mg/mL) (Heng Rui Pharmaceuticals, Jiangsu, China). Patients allocated to WSCM will undergo HSG with ioversol injection, a solution of low osmolar contrast medium that contains iodine $(320 \mathrm{mg} \mathrm{I} / \mathrm{mL}$ ) (Heng Rui Pharmaceuticals). HSG will be performed by a radiologist, without premedication, on the third to seventh day after complete cessation of menstrual bleeding and before the 14th day in the ovulatory cycle.

HSG will be performed as follows: (1) The patient will be placed in supine position. (2) Routine disinfection will be performed in the bladder lithotomy area of the patient. (3) Vaginal speculum will be used to expose the vagina and cervix uteri, then disinfection will be implemented. (4) A rubber double-lumen tube or a special catheter will be inserted into the cervix uteri and will then be fixated. (5) Up to $10 \mathrm{~mL}$ of contrast medium will be slowly injected into the uterus under appropriate pressure until adequate uterine filling has occurred or contrast medium has flowed into the pelvic cavity. This will be directly monitored by fluoroscopy. (6) During the infusion, the dynamic flow of the course of the contrast medium into the uterine cavity and the Fallopian tube will be observed, and images will be captured before the

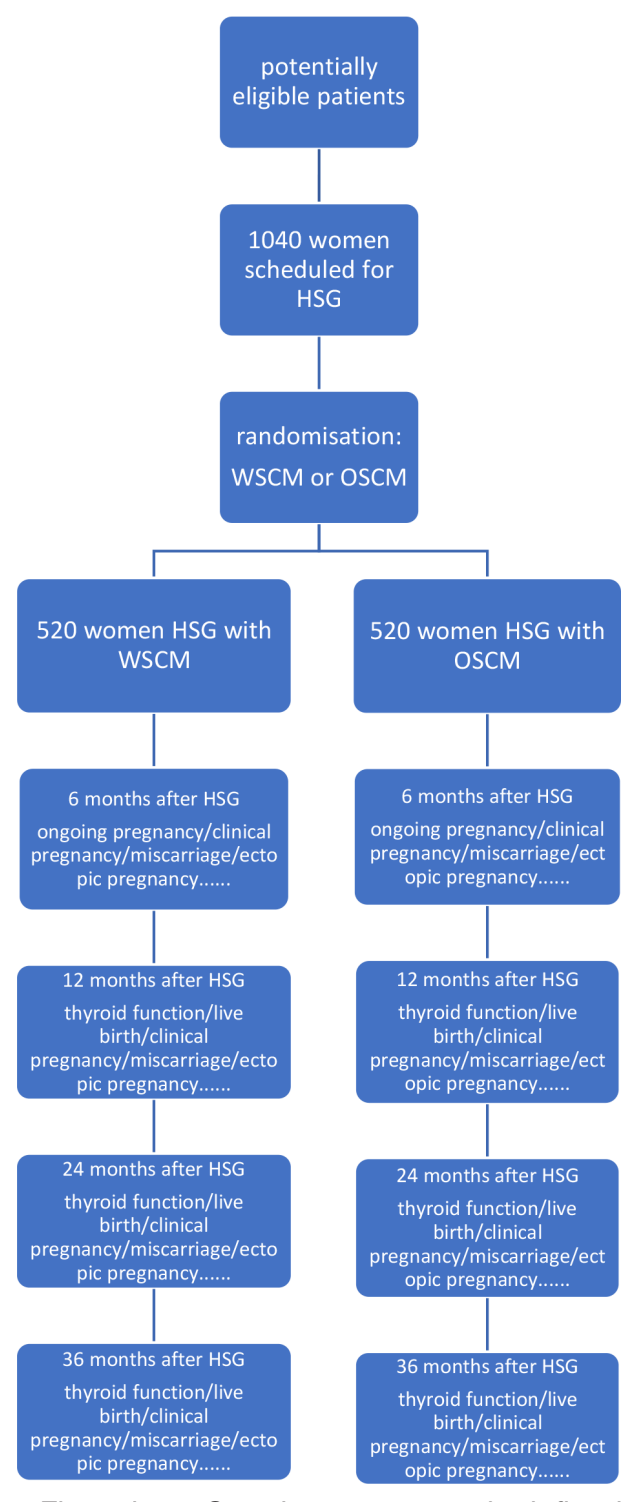

Figure 1 Flow chart. Ongoing pregnancy is defined as a registered heart beat on ultrasound beyond 12 weeks of gestation. Clinical pregnancy is defined as an ultrasoundvisible gestational sac. Miscarriage is defined as a spontaneous loss of pregnancy. Ectopic pregnancy is defined as an embryo implanted outside the uterine cavity. Live birth is defined as the birth of at least one living child. HSG, hysterosalpingography; OSCM, oil-soluble contrast media; WSCM, water-soluble contrast media. 
Table 1 Schedule of activities

\begin{tabular}{|c|c|c|c|c|c|c|}
\hline Procedures & $\begin{array}{l}\text { Screening } \\
\text { Day }-7 \text { to }-1\end{array}$ & $\begin{array}{l}\text { Enrolment/ } \\
\text { baseline } \\
\text { Visit 1, day } 1\end{array}$ & $\begin{array}{l}\text { Study visit } 2 \\
\text { Month } 6\end{array}$ & $\begin{array}{l}\text { Study visit } 3 \\
\text { Month } 12 \\
\end{array}$ & $\begin{array}{l}\text { Study visit } 3 \\
\text { Month } 24 \\
\end{array}$ & $\begin{array}{l}\text { Study visit } 3 \\
\text { Month } 36\end{array}$ \\
\hline Informed consent & $x$ & & & & & \\
\hline Demographics & $x$ & & & & & \\
\hline Medical history & $x$ & & & & & \\
\hline Randomisation & & $x$ & & & & \\
\hline Study intervention & & $x$ & & & & \\
\hline Ongoing pregnancy & & & $x$ & & & \\
\hline Thyroid function of patients & $x$ & & $x$ & $x$ & $x$ & $x$ \\
\hline Neonatal thyroid function & & & & $x$ & $x$ & $x$ \\
\hline $\begin{array}{l}\text { Visual Analogue Scale for pain } \\
\text { scores during the procedure }\end{array}$ & & $x$ & & & & \\
\hline Live birth & & & & $X$ & & X \\
\hline Clinical pregnancy & & & $X$ & & & $X$ \\
\hline Miscarriage & & & $X$ & & & $X$ \\
\hline Ectopic pregnancy & & & $X$ & & & $X$ \\
\hline Safety of intervention & & $X$ & & & & $X$ \\
\hline $\begin{array}{l}\text { Additional therapies (assisted } \\
\text { reproductive technology/ } \\
\text { operation) }\end{array}$ & & & $X$ & & & $X$ \\
\hline Cost calculation & & $X$ & & & & \\
\hline Complete case report files & X & & & & & \\
\hline
\end{tabular}

contrast agent is injected and after the uterine cavity is filled, while the Fallopian tube appears. (7) When the images overlap, the tube or bed position will be changed as needed. After the procedure, images will be reviewed by a radiologist and a diagnosis will be established.

\section{Withdrawal of participants}

Participants will be free to withdraw from the study at any time on request. An investigator may discontinue or withdraw a participant from the study for the following reasons: the participant meets an exclusion criterion, violation of research protocol, and the patient is experiencing an urgent medical situation.

The reasons for participant discontinuation or withdrawal from the study will be recorded on the case report form (CRF). Data collection will continue if data can be safely acquired, and the data will be used for the intentionto-treat (ITT) analyses.

\section{Participant timeline}

The schedule for enrolment, interventions and assessments is summarised in table 1 . The investigators will record the information on the CRF and source documents.

The randomisation and HSG will be performed on day 1. A review of patient information will be done prior to enrolment to determine preliminary eligibility according to the inclusion and exclusion criteria. When a patient provides informed consent, she will be considered for enrolment in the study. Detailed clinical information including age, body mass, duration of infertility, previous surgery and information on her partner will be collected. Follow-up and measurements will be the same for both groups, with a total follow-up of 3 years. Data will be collected at baseline and at 6, 12, 24 and 36 months by telephone follow-up survey or e-questionnaire survey. Procedure steps, pain scores and complications will be recorded. All additional therapies or transfer to other treatments after the first intervention will be recorded in both groups, such as IUI, in vitro fertilisation, intracytoplasmic sperm injection and operation (laparoscopic or hysteroscopic surgery).

\section{Outcome measures}

Primary outcome measures

The primary outcome is the rate of ongoing pregnancy in each treatment group. Ongoing pregnancy will be defined as a registered heart beat on ultrasound beyond 12 weeks of gestation, with the first day of the last menstrual cycle as the beginning of the pregnancy within 6 months after randomisation. 
Secondary outcome measures

- The thyroid function of patients will be examined before HSG, at 4, 8, 12 and 24 weeks, and at 9-12 months after HSG. We will detect free triiodothyronine (FT3), free thyroxine (FT4), thyroid stimulating hormone (TSH), antithyroglobulin antibodies and antithyroid peroxidase antibodies levels at different time points. ${ }^{27} 28$

- The thyroid function of the neonates will be tested within 3-7 days after birth. TT4 (total thyroxine), FT4 and TSH will be detected. ${ }^{28}$

- Pain scores during HSG will be measured by means of the Visual Analogue Scale for pain (scores range from 0.0 to $10.0 \mathrm{~cm}$, with higher scores indicating more severe pain). The pain scores will be recorded by a trained nurse. Meanwhile, the time from the start of contrast injection to the occurrence of pain will be recorded.

- The rates of live birth rate/clinical pregnancy/miscarriages/ectopic pregnancy will be assessed. Live birth is defined as the birth of at least one living child. Clinical pregnancy is defined as an ultrasound-visible gestational sac. Miscarriage is defined as a spontaneous loss of pregnancy. Ectopic pregnancy is defined as an embryo implanted outside the uterine cavity. Each occurrence of one of these events will be recorded during the 3-year follow-up.

- The time to ongoing pregnancy is defined as the time from randomisation to the first day of the last menstrual period plus 4 weeks. It will be considered when ongoing pregnancy occurs. The time to first live birth is defined as the time from randomisation to the date of the first live birth. It will be considered when live birth occurs.

- The costs and cost-effectiveness of OSCM/WSCM and assisted reproductive technology treatments in Chinese context.

- The side effects or complications after therapy in both groups.

\section{Safety assessments}

All adverse events (AEs) will be recorded during the entire study period. An AE is defined as an event during or following HSG or follow-up which was not intended to happen and is suspected to be a complication of the intervention performed. Common AEs of HSG include allergic reactions, artificial abortion syndrome, abdominal pain and intravasation. The severity of the AEs will be primarily ranked as 'mild', 'moderate' or 'severe'. The causes of the AEs will be rated as 'definitely related', 'probably related', 'possibly related', 'probably not related', 'definitely not related' or 'unknown'.

A severe adverse event (SAE) is defined as death, illness necessitating hospitalisation, disability or congenital malformation. All SAEs will be reported within 24 hours to the ethics committee that approved the protocol.

\section{Statistical considerations}

Sample size calculation

In Dreyer $e t a l \mathrm{~s}^{8}$ research, the rate of ongoing pregnancy in the WSCM group was $29 \%$. To detect a difference of $10 \%$ in rates of ongoing pregnancies between the OSCM and the WSCM group, with 1:1 allocation ratio, 90\% statistical power and a two-sided significance level of $5 \%$, we calculated that 467 women per group for a total of 934 will need to be enrolled. Thus, 1040 women (520 in each group) will need to be randomised, including $10 \%$ loss to follow-up or protocol variation.

\section{Statistical analysis}

All data will be analysed according to the ITT principle. SPSS software V.22.0 or higher and R V.3.3.1 (R Project for Statistical Computing, Vienna, Austria) will be used for statistical analyses. Two-sided $p$ values of less than 0.05 will indicate statistical significance.

\section{Statistical analysis of the first 6 months}

As for the primary outcome, we will use the $\chi^{2}$ test to compare the rate of ongoing pregnancy between the OSCM group and the WSCM group. We will use KaplanMeier curves with a log-rank test to compare the difference in time to ongoing pregnancy between the two groups.

We will use the $\chi^{2}$ test to compare the rates of clinical pregnancy, miscarriages and ectopic pregnancy during 6 months after randomisation between the two groups. As for the pain scores during HSG, independent t-test or the Mann-Whitney $U$ test will be used to compare the average pain scores and the mean interval between the start of the contrast injection and the occurrence of pain. ${ }^{29}$

\section{Statistical analysis of the 3-year follow-up}

We will calculate cumulative pregnancy rate of the two groups at 1, 2 and 3 years, and use the $\chi^{2}$ test to assess statistical significance. The cumulative live birth rate will be analysed as the cumulative pregnancy rate. We will use Kaplan-Meier curves with a log-rank test to compare the difference in time to first live birth between the two groups. During the 3-year follow-up, we will record every occurrence of clinical pregnancy or miscarriage or ectopic pregnancy, which may be considered as exploratory outcomes. We may not focus on the multiplicity of type I error on these outcomes. As for the thyroid function of the patients, we will use analysis of variance to compare it at different time points. ${ }^{27}$

Categorical data will be described as absolute numbers and percentages. Distributed continuous variables will be reported as median with IQR.

\section{Subgroup analysis}

To identify a subgroup effect, we plan to test for an interaction for the following subgroups: (1) age of the patients (<35 years, $\geq 35$ years) and (2) primary versus secondary fertility. We may perform some other subgroup analyses when we finally analyse the data. 


\section{Missing data analysis}

We will use multiple imputation to process missing data.

\section{Data monitoring and auditing}

Data monitoring and auditing will be conducted for quality assurance. Monitoring staff will consist of an independent data monitoring committee (IDMC) and an ethics committee. The IDMC is composed of five members, namely one statistician, three clinical experts and one ethicist. They will visit the institutions at important time points throughout the trial, for example, at participant enrolment, at the study interim point and at study completion. Monitoring staff will ensure consistency concerning data documented in both the CRF and the source document and will ensure that the entire study process is in accordance with the approved protocol.

An IDMC and ethics committee will review data trimonthly during the accrual period and near the time that is planned for interim analyses. At each meeting, the IDMC will be asked to give advice on whether the accumulated data from the trial, together with results from other relevant trials, justify continuing recruitment of further patients or further follow-up. An interim analysis on the ongoing pregnancy rate is planned for the time when approximately $50 \%$ participants have been recruited. To control for overall type I error, the stopping boundaries for interim and final analyses will be computed using the Lan-DeMets approximation to the O'Brien-Fleming boundary.

Maintenance of participant confidentiality will involve the following: (1) asking subjects to only share personal and study-related information during our study; (2) storing data in password-protected files on a designated specific computer with restricted access; and (3) only the research-related person will have access to personal identifiable information, which will be destroyed once the study is completed.

\section{Ethics and dissemination}

This protocol received authorisation from the Medical Research Ethics Committee of International Peace Maternity and Child Health Hospital on 18 January 2020 (approval no GKLW2020-02). The findings will be reported in peer-reviewed publications and presentations at international scientific meetings.

\section{Patient and public involvement}

Neither the patients nor the public will be involved in the study design. They will also not be involved in the recruitment process or conduct of the study. The results will be disseminated to patients via an open access publication and our local trials teams.

\section{Trial status}

The recruitment starts from 1 August and we expect to complete the recruitment in August 2021.

\section{DISCUSSION}

HSG is widely used during fertility assessment. Most clinics in China use WSCM, while some use OSCM. Until now, no consensus has been reached on which reagent is better and for which patients. Dreyer et al's research $^{8}$ was the largest RCT on the effects between the two kinds of contrast at HSG; however, it remains unclear if the results apply to the Chinese population. Therefore, the aim of this study is to determine whether using OSCM at HSG results in higher ongoing pregnancy rates compared with using WSCM during 6 months after randomisation, and to explore the difference in maternal and neonatal thyroid function between the two groups.

The strengths of this study are as follows. First, this will be the first study to assess the thyroid function of patients at different time points who undergo HSG and assess their newborns' thyroid function in an RCT. Second, compared with other studies, we will extend the follow-up period to evaluate the long-term impact of HSG using different reagents. Finally, this will be the first record of pain intensity during HSG and the time interval of when pain occurs.

The main shortcomings of this study are that the trial will not be blinded with respect to participants and caregivers due to differences in imaging between OSCM and WSCM and the objectivity of our outcomes. Another limitation is that the trial is based in a single centre, which might limit the generalisability of the findings.

Therefore, we have designed this study to provide rigorous medical evidence for future clinical application of HSG.

\section{Author affiliations}

${ }^{1}$ Department of Obstetrics and Gynecology, International Peace Maternity and Child Health Hospital, School of Medicine, Shanghai Jiao Tong University, Shanghai,

China

${ }^{2}$ Shanghai Key Laboratory Embryo Original Diseases, Shanghai, China

${ }^{3}$ Shanghai Municipal Key Clinical Specialty, Shanghai, China

${ }^{4}$ Department of Radiology, International Peace Maternity and Child Health Hospital, School of Medicine, Shanghai Jiao Tong University, Shanghai, China

${ }^{5}$ Clinical Research Institute, Shanghai Jiao Tong University School of Medicine, Shanghai, China

Acknowledgements The authors thank Elsevier Language Editing Services for polishing the manuscript.

Contributors JZ conceived of the study and participated in its design, as well as supervised the study and critically revised the manuscript. ZQ critically revised the manuscript and contributed to HSG examination. GL participated in writing the manuscript. LJ contributed to HSG examination. QZ and XH contributed to follow-up of patients. $\mathrm{CZ}$ and XW contributed to data collection. LX contributed to revising the statistical methods. All authors read and approved the final version of the manuscript.

Funding This work was supported by the Shanghai Municipal Key Clinical Specialty (shslczdzk01802), International Peace Maternity and Child Health Hospital Clinical Applied Research Program (YN201915).

Competing interests None declared.

Patient and public involvement Patients and/or the public were not involved in the design, or conduct, or reporting, or dissemination plans of this research.

Patient consent for publication Not required.

Provenance and peer review Not commissioned; externally peer reviewed. 
Open access This is an open access article distributed in accordance with the Creative Commons Attribution Non Commercial (CC BY-NC 4.0) license, which permits others to distribute, remix, adapt, build upon this work non-commercially, and license their derivative works on different terms, provided the original work is properly cited, appropriate credit is given, any changes made indicated, and the use is non-commercial. See: http://creativecommons.org/licenses/by-nc/4.0/.

\section{ORCID iDs}

Li Xie https://orcid.org/0000-0003-1892-2217

Jian Zhang https://orcid.org/0000-0003-3664-5807

\section{REFERENCES}

1 Zegers-Hochschild F, Adamson GD, Dyer S, et al. The International glossary on infertility and fertility care, 2017. Fertil Steril 2017;108:393-406.

2 Zhenwu Z, Wenli L. Estimating the prevalence of infertility in China using census data. Population Research 2020;44:3-17.

3 Simpson WL, Beitia LG, Mester J, et al. Hysterosalpingography: a reemerging study. Radiographics 2006;26:419-31.

4 Martí-Bonmatí L. Hysterosalpingography and fertility: a technical relationship. Fertil Steril 2018:110:642.

5 Quaas A, Dokras A. Diagnosis and treatment of unexplained infertility. Rev Obstet Gynecol 2008;1:69-76.

6 Jinxia $B$, Jing $H$, Shengli W. Clinical progress of tubal patency tests. J Int Obstet Gynecol 2020;47:111-4.

7 National Collaborating Centre for Women's and Children's Health (UK. Fertility: Assessment and treatment for people with fertility problems | Information for the public | NICE. Rcog Press 2013;1:21-8.

8 Dreyer K, van Rijswijk J, Mijatovic V, et al. Oil-Based or water-based contrast for hysterosalpingography in infertile women. N Engl J Med Overseas Ed 2017;376:2043-52.

9 Yun AJ, Lee PY. Enhanced fertility after diagnostic hysterosalpingography using oil-based contrast agents may be attributable to immunomodulation. AJR Am J Roentgenol 2004;183:1725-7.

10 Zijun D. Study on clinical effect of loversol and iodized oil in the hysterosalpingography. China \&Foreign Medical Treatment 2018;12:175-7.

11 Sueoka K, Asada H, Tsuchiya S, et al. Falloposcopic Tuboplasty for bilateral tubal occlusion. A novel infertility treatment as an alternative for in-vitro fertilization? Hum Reprod 1998;13:71-4.

12 Lindequist S, Justesen P, Larsen C, et al. Diagnostic quality and complications of hysterosalpingography: oil- versus water-soluble contrast media--a randomized prospective study. Radiology 1991;179:69-74.

13 Johnson NP, Farquhar CM, Hadden WE, et al. The FLUSH trial-flushing with lipiodol for unexplained (and endometriosis-related) subfertility by hysterosalpingography: a randomized trial. Hum Reprod 2004;19:2043-51.

14 Ogata R, Nakamura G, Uchiumi Y, et al. Therapeutic efficacy of hysterosalpingography (HSG) in infertility: a prospective, randomized, clinical study. Jap J Fertil Steril 1993;38:91-4.
15 Johnson JV, Montoya IA, Olive DL. Ethiodol oil contrast medium inhibits macrophage phagocytosis and adherence by altering membrane electronegativity and microviscosity. Fertil Steril 1992;58:511-7.

16 Johnson NP. Review of lipiodol treatment for infertility - an innovative treatment for endometriosis-related infertility? Aust N Z J Obstet Gynaecol 2014;54:9-12.

17 Fang F, Bai Y, Zhang Y, et al. Oil-Based versus water-based contrast for hysterosalpingography in infertile women: a systematic review and meta-analysis of randomized controlled trials. Fertil Steril 2018;110:153-60.

18 Alper MM, Garner PR, Spence JE, et al. Pregnancy rates after hysterosalpingography with oil- and water-soluble contrast media. Obstet Gynecol 1986;68:6-9.

19 de Boer AD, Vemer HM, Willemsen WN, et al. Oil or aqueous contrast media for hysterosalpingography: a prospective, randomized, clinical study. Eur J Obstet Gynecol Reprod Biol 1988;28:65-8.

20 Lindequist S, Rasmussen F, Larsen C. Use of iotrolan versus ethiodized poppy-seed oil in hysterosalpingography. Radiology 1994;191:513-7.

21 Spring DB, Barkan HE, Pruyn SC. Potential therapeutic effects of contrast materials in hysterosalpingography: a prospective randomized clinical trial. Kaiser Permanente infertility work group. Radiology 2000;214:53-7.

22 So S, Yamaguchi W, Tajima $\mathrm{H}$, et al. The effect of oil and watersoluble contrast medium in hysterosalpingography on thyroid function. Gynecol Endocrinol 2017;33:682-5.

23 Miyamoto Y, Tsujimoto T, Iwai K, et al. Safety and pharmacokinetics of iotrolan in hysterosalpingography. retention and irritability compared with lipiodol. Invest Radiol 1995;30:538-43.

24 Satoh M, Aso K, Katagiri Y. Thyroid dysfunction in neonates born to mothers who have undergone hysterosalpingography involving an oil-soluble iodinated contrast medium. Horm Res Paediatr 2015;84:370-5

25 Cooper TG, Noonan E, von Eckardstein S, et al. World Health organization reference values for human semen characteristics. Hum Reprod Update 2010;16:231-45.

26 Coppus SFPJ, Verhoeve HR, Opmeer BC, et al. Identifying subfertile ovulatory women for timely tubal patency testing: a clinical decision rule based on medical history. Hum Reprod 2007;22:2685-92.

27 Kaneshige T, Arata N, Harada S, et al. Changes in serum iodine concentration, urinary iodine excretion and thyroid function after hysterosalpingography using an oil-soluble iodinated contrast medium (lipiodol). J Clin Endocrinol Metab 2015;100:E469-72.

28 Ad hoc writing Committee for guideline on diagnosis and management of thyroid diseases during pregnancy and postpartum (2nd edition); Chinese Society of endocrinology, Chinese medical Association; Chinese Society of perinatology, Chinese medical association. guideline on diagnosis and management of thyroid diseases during pregnancy and postpartum (2nd edition) 2019;22:505-39.

29 van Welie N, Dreyer K, van Rijswijk J, et al. Treatment effect of oil-based contrast is related to experienced pain at HSG: a post-hoc analysis of the randomised H2Oil study. Hum Reprod 2019;34:2391-8. 